\title{
Atividade física e esporte adaptado nos transtornos neurogênicos
}

\author{
Physical activity and adapted sport in the neurogenic disorders
}

\section{Emerson Fachin Martins ${ }^{1}$}

\begin{abstract}
RESUMO
A atividade física assumiu um papel importante no tratamento de transtornos neurogênicos em todos os níveis de atenção à saúde. A prática de atividade física e esporte produz efeitos que são utilizados em programas educativos e terapêuticos com contribuições física, mental, psíquica e sócio-cultural. Os programas de atividade física e esporte adaptado podem ser orientados de maneira a explorar os efeitos terapêuticos da utilização do movimento em modalidades passivas ou ativas de recrutamento neuromuscular. Fatores intrínsecos e extrínsecos às doenças neurológicas devem ser considerados, uma vez que os danos que afetam o sistema nervoso geram perdas funcionais definidos aqui como transtornos que interferem nas habilidades individuais. Para eliminar os impedimentos intrínsecos, programas devem ser estruturados considerando-se o período de manifestação da doença (pré-patológico ou patológico), o caráter progressivo desta manifestação e o conjunto de disfunções isoladas ou combinadas de transtornos sensoriais, motores ou cognitivos. Outros impedimentos, porém agora extrínsecos, que devem ser considerados são obstáculos gerados por locais inapropriados, transportes não adaptados, falta de oportunidades no meio social, acesso a equipamentos adequados e profissionais capacitados. Avaliando-se e considerando-se os fatores intrínsecos e extrínsecos é possível estabelecer programas de atividade física e esporte adaptado que contribuem para o processo de reabilitação.
\end{abstract}

Unitermos: Atividade Motora. Medicina Esportiva. Educação. Terapia por Exercício. Neurologia.

Citação: Martins EF. Atividade física e esporte adaptado nos transtornos neurogênicos.

Trabalho realizado na Universidade Universidade de Brasília (UnB).

1.Fisioterapeuta, Doutor em Neurociência e Comportamento pela USP, Professor Adjunto da Faculdade de Ceilândia (FCE) da Universidade de Brasília (UnB).

\section{SUMMARY}

Physical activity assumed an important role in the handling of neurogenic disorders in all levels of health attention. Physical activity and sport produce effects that are utilized in therapeutic and educational programs with socio-cultural, psychological, mental, and physical contributions. Physical activity programs and adapted sports can be oriented to explore therapeutics effects using movement in passive or active modalities of neuromuscular recruitment. Intrinsical and extrinsical factors to neurological disease must be considered whereas neurological injuries affecting nervous system generate functional impairments defined here for disorders that they interfere in individual skills. To eliminate intrinsically obstructions, programs must be structured leading in consideration the period of illness manifestation (prepathologic or pathologic), the progressive character of this manifestation and sensorial, motor or cognitive disorders that can be present in isolated or grouped forms. Other, but now extrinsical obstructions, to consider are obstacle caused by inappropriate places, non-adapted transports, absence of opportunities in social environment, access to appropriated equipment and qualified professionals. Evaluating and considering intrinsical and extrinsical factors, its possible establish physical activities programs and adapted sports to contribute for rehabilitation process.

Keywords: Motor Activity. Sports Medicine. Education. Exercise Therapy. Neurology.

Citation: Martins EF. Physical activity and adapted sport in the neurogenic disorders.

Endereço para correspondência: Emerson F Martins Faculdade de Ceilândia (FCE) Universidade de Brasília (UnB) Campus de Ceilândia

QNN 14 Área Especial - Ceilândia Sul 72220-140 Brasília, DF E-mail: efmartins@unb.br

Recebido em: 09/04/07 Revisado em: 10/04/07 a 25/07/07 Aceito em: 26/07/07 Conflito de interesses: não 


\section{INTRODUÇÃO}

A atividade física com finalidade educacional e terapêutica já é descrita por séculos e seu uso em diferentes condições de incapacidade é prática comumentedesenvolvidaporprofissionaisdasaúde ${ }^{1,2}$.

As primeiras concepções de atividade física adaptada vieram com o modelo educacional dos anos 70 que propunha um modelo alternativo aos princípios médicos que utilizavam programas de exercícios com natureza preventiva, desenvolvimentista e corretiva'.

Diferente da chamada ginástica médica que tinha por objetivo prevenir doenças e promover o vigor da mente e corpo, o modelo educacional recomendava que as competências fossem compatíveis com o potencial do indivíduo a ser incluído no programa de atividade física adaptada. Desta forma, enfatizavam-se as condições de utilização das funções remanescentes e não a deficiência'.

Estas primeiras concepções para uso da atividade física em populações especiais convergem com muitas abordagens fisioterapêuticas para reabilitação de disfunções motoras que exploram o potencial funcional da movimentação ativamente acionada após um dano específico ${ }^{3}$.

Somando-se as primeiras concepções médicas, que constitui a origem da atividade física adaptada, com o modelo educacional e as necessidades sociais de fomentar os direitos e oportunidades iguais para populações com necessidades especiais, surge a necessidade de preparar profissionais para a utilização da atividade física nestas condições.

Nos últimos anos, a literatura científica nacional começa a apresentar uma série de trabaIhos mostrando a utilização da atividade física em ações terapêuticas ${ }^{4,5}$, bem como em programas de atividade física e esporte adaptado para diferentes transtornos neurogênicos ${ }^{6-8}$.

O conceito de transtorno neurogênico que será utilizado nesta revisão incorpora as definições sugeridas por Kisner et al. ${ }^{2}$ para se referir às consequências de um comprometimento na estrutura e/oufunção de sistemasorgânicosquesão potencialmente geradores de limitação funcional, incapacidades e deficiências. Quando com origem no sistema nervoso, devido às características de seu funcionamento, acabam por gerar transtornos que podem serclassificadoscomo disfunções sensoriais, motores e/ou cognitivos ${ }^{9}$.
Desta forma, a presente revisão pretende discutir informações que fundamentem o uso da atividade física e esporte adaptado para populações com transtorno neueogênicos de maneira a fornecer subsídios para a elaboração de programas de atividade física específicos para estas condições.

\section{MÉTODO}

Nesta revisão da literatura foram selecionados livros e artigos publicados em fontes nacionais e internacionais sobre o assunto, indexados nas bases de dados: Medline, SciELO, Lilacs, Web of Science e EBSCO.

Nas buscas foram utilizadas as palavras-chaves como atividade motora, medicina esportiva, educação, terapia por exercícios, neurologia, dentre outros sinônimos para localização de referências que tratassem do assunto nos últimos 30 anos.

As referências incluídas foram aquelas que apresentavam informações relevantes para a discussão dos subtemas propostos no desenvolvimento desta revisão. As demais referências foram excluídas.

\section{RESULTADOS}

\section{Transtornos Neurogênicos}

Em função da organização morfofuncional do sistema nervoso, os transtornos manifestados nas doenças de origem neural podem ser classificados como sensoriais, motores e cognitivos 9 .

As disfunções sensoriais manifestam-se por sinais e sintomas gerados por dano na estrutura ou funcionamento dos elementos nervosos que permitem o indivíduo sentir o ambiente que o cerca (exterocepção) e seu próprio corpo (propriocepção e interocepção) ${ }^{10}$. Desta forma, qualquer agressão que altere o funcionamento do receptor sensorial, seu trajeto pelos nervos do sistema nervoso periférico ou pelos tractos do sistema nervoso central irá resultar em transtorno de natureza sensorial.

Da mesma maneira, as disfunções motoras são ocasionadas por sinais e sintomas decorrentes de lesão estrutural ou funcional em elementos neurais do sistema nervoso responsáveis pela resposta motora voluntária e reflexa (movimentação) ou autonômica (neurovegetativa) ${ }^{10}$. Entretanto, diferente das manifestações sensoriais que são ocasionadas unicamente por dano em estruturas 
neurais relacionadas com o envio de informações aferentes exteroceptivas, proprioceptivas e interoceptivas; as manifestações motoras podem ocorrer pela falta desta informação sensorial, mesmo não havendo qualquer lesão sediada em estruturas neurais do sistema motor, visto que tais informações são processadas e integradas para a elaboração das respostas motoras pelo sistema ${ }^{11}$.

Também com natureza mais integrativa de processamento neural, os transtornos geradores de disfunções cognitivas são determinados por danos localizados em estruturas encefálicas que são responsáveis por funções mentais como percepção, atenção, memória, aprendizagem, linguagem, emoções, dentre outras ${ }^{12}$.

Os transtornos neurogênicos podem apresentar-se com comprometimentos geradores de disfunções exclusivamente sensoriais, motores ou cognitivas; contudo, manifestações combinadas são comuns e devem ser consideradas na determinação das estratégias de intervenção educativas e terapêuticas 9 .

\section{Manifestação e progressão das doenças neurológicas}

As doenças que afetam o sistema nervoso podem ser intrínsecas ao sistema, sendo definidas como primárias, ou extrínsecas quando secundárias a doenças que afetam outros sistemas ${ }^{13}$. Desta forma, a identificação do mecanismo de lesão é tão importante quanto à origem das doenças, pois irá determinar a transitoriedade e a progressão da agressão ao sistema nervoso que por sua vez implicará no quadro de disfunções manifestadas 9 .

Assim, qualquer intervenção em indivíduos portadores de transtornos neurogênicos deve levar em consideração qual período de manifestação da doença. É possível identificar dois períodos relacionados a uma moléstia: (1) período pré-patológico caracterizado pela interação entre os agentes mórbidos, hospedeiro humano e os fatores ambientais que possam ocasionar risco de desenvolvimento de uma determinada afecção e (2) período patológico que é iniciado pelas primeiras alterações após adquirido uma doença e que determinarão o quadro clínico de disfunções ${ }^{14}$.

No período pré-patológico as intervenções constituem-se por ações preventivas e de promoção da saúde que pretendem eliminar o risco do indivíduo vir a manifestar uma determinada afec- ção. Já no período patológico, as ações visam inibir, minimizar ou controlar a progressão da doença instalada ${ }^{14}$.

Assim, o caráter gradual da manifestação das disfunções na doença é um bom critério para classificação quando utilizada para determinação de programas de atividade física por profissionais que tem como objetivo intervir com ações educativas e terapêuticas agrupando as doenças neurológicas em progressivas e não progressivas.

As doenças progressivas representam aquelas cujos processos fisiopatológicos provocam danos contínuos no sistema nervoso e, à medida que a lesão expande, aumenta gradativamente as disfunções e seqüelas manifestadas. São exemplos de doenças neurológicas progressivas a Doenças de Parkinson, a Esclerose Múltipla, a Esclerose Lateral Amiotrófica ${ }^{4,9,12-14}$.

Já nas doenças não progressivas, o agente agressor promove o dano primário no sistema nervoso que determinará as disfunções e seqüelas relacionadas à área lesada como é comumente observado em doenças por malformações ou lesões congênitas, moléstias cerebrovasculares e traumatismos cranianos ou raquimedulares 9,12-14.

\section{Programas de atividade física para Transtornos Neurogênicos}

Aspectos multifatoriais acabam por influenciar a aplicação de programas de atividade física como ações educativas e terapêuticas em portadores de doenças neurológicas ${ }^{15}$.

Aspectos físicos são determinados pela interação de fatores individuais e ambientais que influenciam a capacidade de coordenar movimentos para uma determinada prática ou treinamento. Tal coordenação motora é uma habilidade adquirida após milhões de etapas em processo de aprendizagem durante a infância que é aperfeiçoada por toda a vida ${ }^{16}$.

Tais aspectos físicos são diretamente influenciados pela capacidade cognitiva do indivíduo que permite a harmonia entre ações reflexas, automáticas e voluntárias na composição da movimentação funcional humana e determinam a habilidade de reproduzir um programa de atividade motora ${ }^{17}$.

Ainda, aspectos físicos e mentais são influenciados por estados motivacionais e emocionais também relacionados aos determinantes sócio-culturais que apresenta um estigma diver- 
gente das características encontradas no portador de deficiência de que um corpo forte e sadio seja requisito para a prática de atividade física ${ }^{15}$.

Se admitirmos que o sistema nervoso é o grande responsável pelo controle das funções motoras e mentais e, ainda, pela capacidade cognitiva de um indivíduo, fica claro que a prática de atividade física para indivíduos portadores de transtornos neurogênicos necessita de acompanhamento de profissional que saiba reconhecer as manifestações decorrentes da lesão no sistema nervoso de maneira a estabelecer programas que potencialize as capacidades do indivíduo pela estimulação adequada de um sistema nervoso com alterações de seu funcionamento.

A prática de atividade física em seres humanos onde todos os pré-requisitos para a produção de movimento estão presentes, produz atividade motora coordenada que é efetivamente alcançada pela repetição do movimento e pela exploração do ambiente ${ }^{18}$.

Entretanto, em portadores de transtornos neurogênicos, habilidades motoras básicas estão perdidas, tornando necessário o treinamento funcional de um repertório mínimo de funções motoras e a utilização de estratégias que permitam uma nova aprendizagem de como se movimentar, capacitando-os ao programa de atividade física proposto $^{19}$.

Partindo desta premissa, a atividade física indicada para esta população poderia ser considerada em uma seqüência de duas abordagens. A abordagem primária considera objetivos mais terapêuticos que são voltados à reabilitação e desenvolvimento de capacidades funcionais que seriam utilizadas em uma abordagem secundária com finalidades mais educativas que são próprias da prática de esporte (Figura 1).

$\mathrm{Na}$ abordagem inicial, com finalidades terapêuticas, os programas de atividade física para pessoas com transtornos neurogênicos podem ser graduados progressivamente conforme conceitos fisioterapêuticos em programas que evoluem de uma movimentação mais passiva para formas mais ativas ${ }^{2}$.

Os programas com movimentação passiva são aqueles em que a produção do movimento se dá por uma força externa gerada por outro indivíduo ou equipamento mecânico e seus objetivos estão relacionados à amplitude de movimento articular ${ }^{20,21}$.
Para programas com movimentação ativa surgem sub-classificações que levam em conta o aspecto gradativo de participação voluntária denominadas de programas ativo-assistido, ativo-livre e ativo-resistido $^{2}$.

Também com objetivos relacionados à amplitude de movimento articular, porém com algum grau de estimulação muscular, os programas ativos-assistidos são caracterizados pelo movimento produzido por uma força externa que auxilia um indivíduo no recrutamento muscular ativo necessário para a realização de uma atividade motora ${ }^{2}$.

Já nos programas ativo-livre e ativo-resistido não existe contribuição de força externa para a produção de movimento sendo realizado recrutamento muscular voluntário livre somente resistido pela ação da gravidade ou contra-resistido por carga adicional à ação gravitacional, respectivamente ${ }^{2}$. Nestes programas a natureza da atividade física permite objetivos relacionados ao condicionamento cardiorrespiratório e muscular, além de benefícios osteomioarticulares com grandes repercussões terapêuticas ${ }^{22-24}$.

Depois de adquirido um repertório mínimo de habilidade motora por meio de programas bastante específicos e com objetivos terapêuticos como os mencionados acima, os portadores de transtornos neurogênicos tornam-se capazes de realizar outros programas de atividade física com finalidades mais educativas do que terapêuticas e podem ser direcionadas a programas que explorem o potencial destes indivíduos por meio de esporte adaptado.

\section{Atividade física e esporte adaptado para condições neurológicas}

A atividade física e o esporte adaptado, além de terem como objetivos o lazer e a competição, são considerados aceleradores do processo de reabilitação ${ }^{15}$. Encontra-se a seguir uma série de relatos que descrevem os benefícios e implicações desta prática para indivíduos acometidos por transtornos de origem neurológica.

Os fatores que influenciam na adesão de deficientes visuais e motores para prática esportiva são os fatores extrínsecos à deficiência, que apresentaram os maiores empecilhos para a prática de atividade física adaptada ${ }^{25}$.

Eliminados os impedimentos extrínsecos à deficiência como profissional especializado, transporte, local adaptado, desporto adaptado, condi- 
Figura 1. A abordagem seqüencial de indicação de atividade física e esporte adaptado que evolui de indicações com finalidades mais terapêuticas para indicações com finalidades mais educativas. Ainda, apresentam-se os efeitos desejados por cada indicação.

\begin{tabular}{|c|c|c|c|c|}
\hline \multirow{2}{*}{$\begin{array}{l}\text { TRANSTORNO NEURO- } \\
\text { GÊNICO }\end{array}$} & \multicolumn{2}{|c|}{ ATIVIDADE FÍSICA E ESPORTE ADAPTADO } & \multicolumn{2}{|c|}{ EFEITOS ESPERADOS PELA INDICAÇÃO } \\
\hline & Abordagem Primária & Abordagem Secundária & Finalidade Terapêutica & Finalidade Educativa \\
\hline SENSORIAIS & $\begin{array}{l}\text { 1. Programa de movi- } \\
\text { mentação ativa-assis- } \\
\text { tida². } \\
\text { 2. Programa de movi- } \\
\text { mentação ativa livre } 2 \text {. } \\
\text { 3. Programa de } \\
\text { movimentação ativa } \\
\text { resistida2. }\end{array}$ & $\begin{array}{l}\text { 1. Atividade física adaptada } \\
\text { para cegos não especificada } \\
\text { pelo autor }{ }^{25} \text {. } \\
\text { 2. Voleibol e Natação adapta- } \\
\text { dos para surdos }{ }^{15} \text {. } \\
\text { 3. Esportes adaptados para } \\
\text { cegos chancelados pela } \\
\text { Associação Brasileira de } \\
\text { Desporto para Cegos }{ }^{15} \text {. }\end{array}$ & $\begin{array}{l}\text { 1. Garantir integridade } \\
\text { osteomioarticular promo- } \\
\text { vendo flexibilidade, ativação } \\
\text { muscular e aquisição de } \\
\text { repertório mínimo de movi- } \\
\text { mentos para treinamentos } \\
\text { funcionais futuros. } \\
\text { 2. Promover condiciona- } \\
\text { mento cardiovascular e } \\
\text { respiratório e estimular a } \\
\text { coordenação motora em } \\
\text { seqüências de treinamento } \\
\text { funcional para aquisição de } \\
\text { repertório mínimo de movi- } \\
\text { mentos para atividade física } \\
\text { e esportes adaptados. } \\
\text { 3. Promover força e resistên- } \\
\text { cia muscular. }\end{array}$ & $\begin{array}{l}\text { 1. Inclusão social e po- } \\
\text { pularização do desporto } \\
\text { adaptado } \\
\text { 2. Inclusão social, auto- } \\
\text { conhecimento de suas po- } \\
\text { tencialidades e desenvol- } \\
\text { vimento físico, cognitivo, } \\
\text { afetivo e social }{ }^{15} \text {. } \\
\text { 3. Efeitos já mencionados } \\
\text { nos itens acima acrescidos } \\
\text { da formação de atributos } \\
\text { como perseverança, inicia- } \\
\text { tiva, prudência e domínio } \\
\text { próprio }{ }^{15} \text {. }\end{array}$ \\
\hline MOTORES & $\begin{array}{l}\text { 1. Programa de movi- } \\
\text { mentação passiva². } \\
2 \text { a } 4 \text {. Fazem referência } \\
\text { aos itens de } 1 \text { a } 3 \text { descri- } \\
\text { tos para os transtornos } \\
\text { sensoriais. }\end{array}$ & $\begin{array}{l}\text { 1. Atividade física adaptada } \\
\text { para deficientes físicos não } \\
\text { especificados pelo autor }{ }^{25} \text {. } \\
\text { 2. Basquetebol e Handebol } \\
\text { adaptado em cadeira de } \\
\text { rodas }^{26} \text {. } \\
\text { 3. Atividade física adaptada } \\
\text { para portadores de Paralisia } \\
\text { Cerebral } 15,28 \text {. } \\
\text { 4. Modalidades de desporto } \\
\text { específicas para cegos }^{27} \text {. }\end{array}$ & $\begin{array}{l}\text { 1. Reverter limitações da } \\
\text { amplitude de movimento } \\
\text { garantindo flexibilidade } \\
\text { necessária para os progra- } \\
\text { mas de movimentação com } \\
\text { envolvimento mais ativo. } \\
2 \text { a } 4 \text {. Fazem referência aos } \\
\text { itens de } 1 \text { a } 3 \text { descritos para } \\
\text { os transtornos sensoriais. }\end{array}$ & $\begin{array}{l}\text { 1. Inclusão social e po- } \\
\text { pularização do desporto } \\
\text { adaptado } \\
\text { 2. Promoção do bem estar } \\
\text { físico e psicológico, aqui- } \\
\text { sição de identidade social, } \\
\text { compromisso individual } \\
\text { e com o grupo, dentre } \\
\text { outras }{ }^{26} \text {. } \\
\text { 3. Efeitos já mencionados } \\
\text { nos itens acima.4. Efeitos já } \\
\text { mencionados nos itens aci- } \\
\text { ma acrescidos do caráter } \\
\text { competitivo }{ }^{27} \text {. }\end{array}$ \\
\hline COGNITIVOS & $\begin{array}{l}\text { 1. Quando associados a } \\
\text { transtornos sensoriaise/ } \\
\text { ou motores, necessitam } \\
\text { da mesma abordagem } \\
\text { descrita acima. } \\
\text { 2. Quando não associa- } \\
\text { dos, indica-se avançar } \\
\text { para a abordagem } \\
\text { secundária. }\end{array}$ & $\begin{array}{l}\text { 1. Atividade física adaptada } \\
\text { para deficientes mentais }{ }^{15} \text {. }\end{array}$ & $\begin{array}{l}\text { 1. Efeitos já mencionados } \\
\text { nos itens acima. }\end{array}$ & $\begin{array}{l}\text { 1. Desenvolvimento físico, } \\
\text { cognitivo e emocional }{ }^{15} \text {. }\end{array}$ \\
\hline
\end{tabular}

ção financeira e material adequado; a atividade física adaptada poderia proporcionar aos deficientes oportunidades de utilizarem suas habilidades residuais para participarem de jogos e desporto com finalidade de desenvolverem o máximo de suas capacidade ${ }^{25}$.

O maior impedimento para a prática do handebol adaptado para portadores de deficiência motora não são as limitações da deficiência, mas sim dificuldades como locais apropriados, transportes adaptados, oportunidades no meio social, acesso a equipamentos adequados e profissionais capacita$\operatorname{dos}^{26}$

O principal fator a se considerar na prática adaptada do voleibol para surdos é a comunica- ção ${ }^{15}$. Assim sendo, uma vez estabelecidas estratégias como escolha de tarefas adequadas com explicações claras e simples, enfatizado o progresso dos alunos por meio de linguagem de domínio deles, disposto o grupo em semicírculo para acentuar o acesso visual utilizando diferentes formas de comunicação por sinais e gestos técnicos; os impedimentos para a prática desta atividade para portadores de surdez seriam eliminados.

Já para deficientes visuais, a prática de atividade física requer a eliminação das informações visuais fazendo com que o profissional deva se atentar ao uso de ações sem a prévia informação da ocorrência delas, ou seja, há a necessidade de se comunicar ações das mais simples como a apro- 
ximação do grupo e detalhamento do ambiente de forma não visual com informações auditivas e

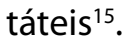

A população de deficientes visuais, além de contar com esportes adaptados, hoje pode utilizar esportes especificamente criados para cegos em que sua prática é totalmente desenvolvida sem a necessidade de informação visual com fornecimento sonoro e tátil que formam um referencial espacial para o praticante ${ }^{27}$.

Para indivíduos portadores de transtornos mais motores que sensoriais, a atividade competitiva em portadores de Paralisia Cerebral, além de ensinar a ultrapassar limites estimula o desenvolvimento motor e social, possibilitando aos praticantes o reconhecimento de suas aptidões e a percepção de que são capazes de realizar atos motores que não sabiam que fossem possíveis de serem realizados ${ }^{28}$.

Entretanto, há evidências de que futebolistas altamente treinados portadores de Paralisia Cerebral apresentam assimetria de forças, fraqueza do músculo quadríceps e desequilíbrio entre músculos antagonistas do joelho, que são fatores de risco para lesão dos joelhos ${ }^{29}$. Tais fatores de risco poderiam ser minimizados por um programa de avaliação e fortalecimento muscular direcionado para esta população ${ }^{29}$.

Há evidências de que o envolvimento de portadores de deficiência neurológica com a natação adaptada promove benefícios não somente para sua melhora físico, mas também para o seu estado emocional influenciando sua qualidade de vida ${ }^{8}$.

\section{CONCLUSÃO}

Evidências na literatura científica têm apontado para muitos benefícios advindos da prática de atividade física e esporte adaptado para portadores de transtornos neurogênicos, porém tais programas necessitam de atenção especial para fatores intrínsecos e extrínsecos à doença que devem eliminar os impedimentos gerados pela deficiência e enfatizar as potencialidades do indivíduo.

\section{REFERÊNCIAS}

1.Sherrill C. Adapted physical activity, recreation and sport: crossdisciplinary and lifespan. 5th ed.. Dubuque: McGraw Hill, 1998, 706p.

2.Kisner C, Colby LA. Therapeutic exercise: foundation and techniques. 5th ed. Philadelphia: FA Davis Company, 2002, 864p.
3.O'Sullivan S, Schmitz TJ. Fisioterapia: avaliação e tratamento. 4a. ed. São Paulo: Manole, 2003, 1200p.

4.Martins EF, Christofani JS. Repetição planejada de padrões funcionais de movimentação modifica a resposta motora e metabólica à fadiga manifestada por pacientes portadores de Esclerose Múltipla. Rev Bras Cienc da Saúde 2006;10:5-15.

5.Calil SR, Santos TABP, Braga DM, Labronici RHDD. Reabilitação por meio da dança: uma proposta fisioterapêutica em pacientes com seqüela de AVC. Rev Neurocienc 2007;15:195-202.

6.Labronici RHDD, Cunha MCB, Oliveira ASB, Gabbai AA. Esporte como fator de integração do deficiente físico na sociedade. Arq Neuropsiquiatr 2000;58:1092-9.

7.Martin JH. Neuroanatomy: text and atlas. 2nd ed. Connecticut: Apleton \& Lange, 1996, 578p.Brazuna MR, Mauerberg-de-Castro E. A trajetória do atleta portador de deficiência física no esporte adaptado de rendimento. Uma revisão da literatura. Motriz 2001;7:115-23.

8.Tsutsumi O, Cruz VS, Chiarello B, Junior DB, Alouche SR. Os benefícios da natação adaptada em indivíduos com lesões neurológicas. Rev Neurocienc 2004;12:82-6.

9.Levy JA, Oliveira ASB. Reabilitação em doenças neurológicas: guia terapêutico prático. São Paulo: Atheneu, 2004, 263p.

10.Kandel ER, Schwartz JH, Jessell TM. Essentials of neural science and behavior. Connecticut: Simon \& Schuster Company, 1995, 743p.

11.Lent R. Cem bilhões de neurônios: conceitos fundamentais de neurociência. São Paulo: Atheneu, 2002, 698p.

12.Rowland LP. Merritt: tratado de neurologia. 10a. ed. Rio de Janeiro: Guanabara Koogan, 2002, 887p.

13.Pereira MG. Epidemiologia: teoria e prática. Rio de Janeiro: Guanabara Koogan, 1995, 234p.

14.Duarte E, Lima SMT. Atividade física para pessoas com necessidades especiais: experiências e intervenções pedagógicas. Rio de Janeiro: Guanabara Koogan, 2003, 104p.

15.Kottke FJ, Halpern D, Easton JKM, Ozel AT, Burrill CA. The training of coordination. Arch Phys Med Rehabil 1978;59:567-2.

16.Shumway-Cook A, Woollacott MH. Controle Motor: teoria e aplicações práticas. Segunda edição. São Paulo: Manole, 2003, 592p.

17.Gallahue D. Motor development. Second Edition. Indianapolis: Brown \& Benchmark, 1989, 382p.

18.Durward BR, Baer GD, Rowe PJ. Movimento funcional humano: mensuração e análise. São Paulo: Manole, 2001, 233p.

19.Clarkson HM, Gilewich GB. Musculoskeletal assessment: joint range of motion and manual muscle strength. Baltimore: Williams \& Wilkins, 1989, 352p.

20.Frank C. Physiology and therapeutic value of passive joint motion. Clin Ortopedic 1984;185:113.

21.Barnes W. Relationship between motor unit activation to muscular contraction at different contractile velocities. Phys Ther 1980;60:1152. 22.Bruce RA. Exercise testing for ventricular function. N Engl J Med 1977;296:671-5.

23.Kakebeeke TH, Roy SH, Largo RH. Coordination training in individual with incomplete spinal cord injury: consideration of motor hierarchical structures. Spinal Cord 2006;44:7-10.

24.Fatores que influenciam a adesão de deficientes motores e deficientes visuais a prática desportiva (endereço na Internet). Buenos Aires: Moreira WC, Rebelo RJ, Paula AH, Cotta DO. Revista Digital 2007;11(104) (atualização: 01/2008; citado em: 01/2009). Disponível em: http://www.efdeportes.com.

25.Esporte adaptado construído a partir das possibilidades: handebol adaptado. (endereço na Internet). Buenos Aires Itani DE, Araújo PF, Almeida JJG. Revista Digital 2004;10(72) (atualização: 01/2009; citado em: 01/2009). Disponível em: http://www.efdeportes.com.

26.Goalball (endereço na Internet). Brasil: Confederação brasileira de desportos para cegos (atualização: 01/2008; citado em 01/2009). Disponível em: http://www.cbdc.org.br.

27.Souza JV. A terapia esportiva na paralisia cerebral: estudo de 133 pacientes. (dissertação). São Paulo: USP, 2002, 112p.

28.Andrade MS, Fleury AM, Silva AC. Força muscular isocinética de jogadores de futebol da seleção paraolímpica brasileira de portadores de paralisia cerebral. Rev Bras Med Esporte 2005;11:281-5. 